Bulletin of the AAS • Vol. 53, Issue 2

\title{
On Constraining \\ Circumgalactic Pressure
}

\section{G. Mark Voit ${ }^{1}$, Ben Oppenheimer ${ }^{2}$}

${ }^{1}$ Michigan State University, ${ }^{2}$ CASA, U. Colorado Boulder

Published on: Dec 31, 2021

DOI: $10.3847 / 25 c 2 c f e b .90562 b 74$

License: Creative Commons Attribution 4.0 International License (CC-BY 4.0). 


\section{from $\underline{\text { Galactic Atmospheres: Perspectives }}$}

The first pressure measurement of a galactic atmosphere came in 1956, and it was indirect. Lyman Spitzer inferred the pressure of hot gas in the Milky Way's halo from absorption-line observations of cold clouds not far above our galaxy's disk [1].

Six and a half decades later, the pressure of the Milky Way's halo at greater distances remains poorly constrained, but that is about to change. The community studying galactic atmospheres has developed diverse observational approaches for assessing circumgalactic pressure that are complementary. Constraints emerging from those approaches can therefore be combined to provide much stronger constraints.

The key to success in this task will be a descriptive parametric model for the circumgalactic medium (CGM). Focusing on an efficient subset of parameters that describes how CGM pressure profiles depend on radius and halo circular velocity will enable maximum likelihood methods to obtain joint constraints on those parameters.

Consider the following simple, purely descriptive parametric model for CGM pressure profiles:

$$
\mathrm{P}\left(\mathrm{r}, \mathrm{v}_{\mathrm{c}}\right)=\mathrm{P}_{100}\left(\frac{\mathrm{v}_{\mathrm{c}}}{200 \mathrm{~km} \mathrm{~s}^{-1}}\right)^{\zeta}\left(\frac{\mathrm{r}}{100 \mathrm{kpc}}\right)^{-\alpha}\left[1+\left(\frac{\mathrm{r}}{\mathrm{r}_{\mathrm{c}}}\right)^{2}\right]^{-(3 \beta-\alpha) / 2}
$$

There are five free parameters describing the pressure $(\mathrm{P})$ of a galactic atmosphere at a particular radius $\mathrm{r}$ in a galactic halo of maximum circular velocity $\mathrm{v}_{\mathrm{c}}$ :

- a normalization factor $\left(\mathrm{P}_{100}\right)$

- a power-law dependence $(\zeta)$ on $\mathrm{v}_{\mathrm{c}}$

- an inner power-law slope $(\alpha)$

- an outer power-law slope parameter $(\beta)$ with heritage in the extensive literature on galaxy groups and clusters

- a transitional "core" radius $\left(\mathrm{r}_{\mathrm{c}}\right)$ at which the pressure profile changes slope

The model expresses CGM pressure as a function of halo circular velocity rather than halo mass, because a halo's circular velocity remains relatively constant with time, whereas the usual definitions of halo mass cause it to increase gradually with time, even if the halo itself is not changing [2] . 
For the same reason, the model expresses CGM pressure as a function of physical radius, rather than scaling it relative to a virial radius. Otherwise, observed radii must be translated into scaled radii using a halo model, which introduces spurious modeldependent covariances into all quantities that are functions of radius.

The time for adopting such a consensus community-spanning model has now come, because complementary constraints on CGM pressure are rapidly accumulating.

For example, constraints on the Milky Way's CGM can be obtained from X-ray absorption, X-ray emission, pressures of atomic hydrogen clouds, ram-pressure stripping of dwarf galaxies, and the dispersion measures of pulsars in the Large Magellanic Cloud []].

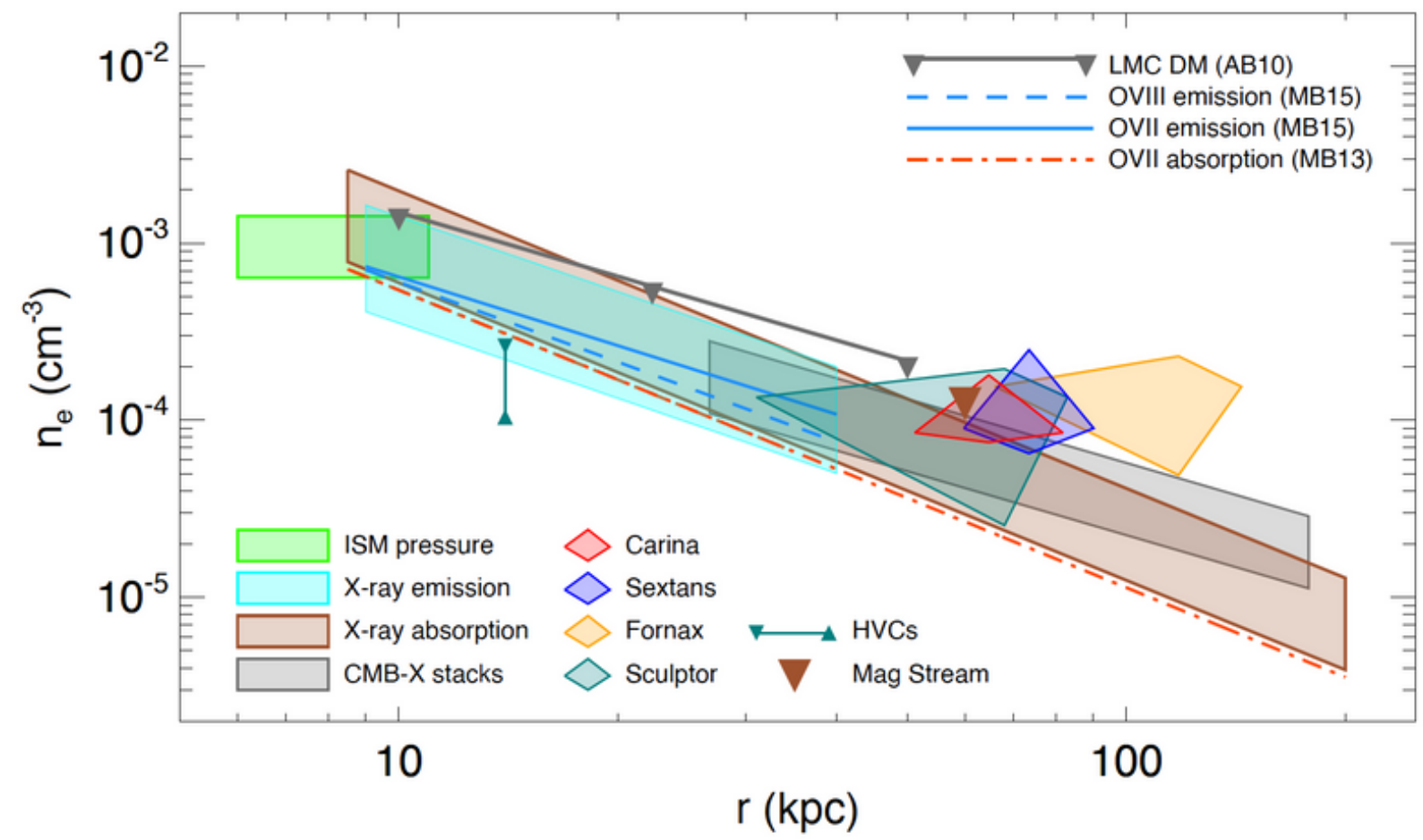

Figure 1

A collection of constraints on the Milky Way's CGM density profile, adapted from Voit (2019). See that paper for details and citations to the data sources.

Constraints on the pressures of $10^{4} \mathrm{~K}$ CGM clouds around other galaxies can be obtained from photoionization modelling. And those constraints have already revealed dependences on circular velocity and radius [4]]. 


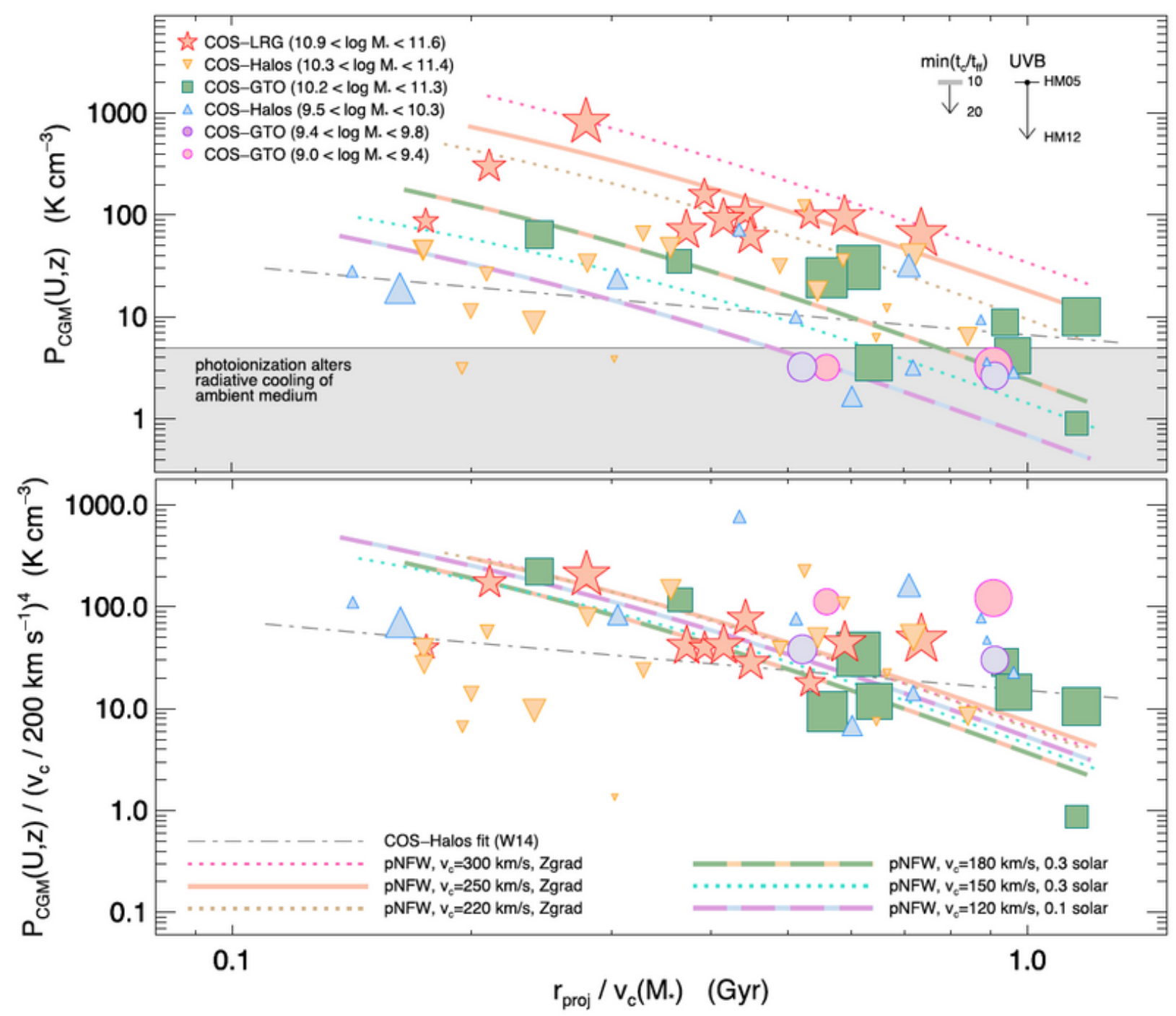

Figure 2

Pressures inferred from photoionization modeling of CGM clouds presumably embedded in hotter gas (see Voit et al. 2019 for details). The top panel shows a clear dependence of CGM pressure on galactic stellar mass. In the bottom panel the implied dependence on halo circular velocity is removed, revealing a common dependence on radius.

Stacking of X-ray data from the ROSAT All-Sky Survey has provided useful constraints on how CGM luminosity depends on galactic stellar mass []ㅡ. In the near future, stacking of eROSITA observations will provide much more detailed information about how CGM surface brightness depends on both radius and halo circular velocity [ [6]. 


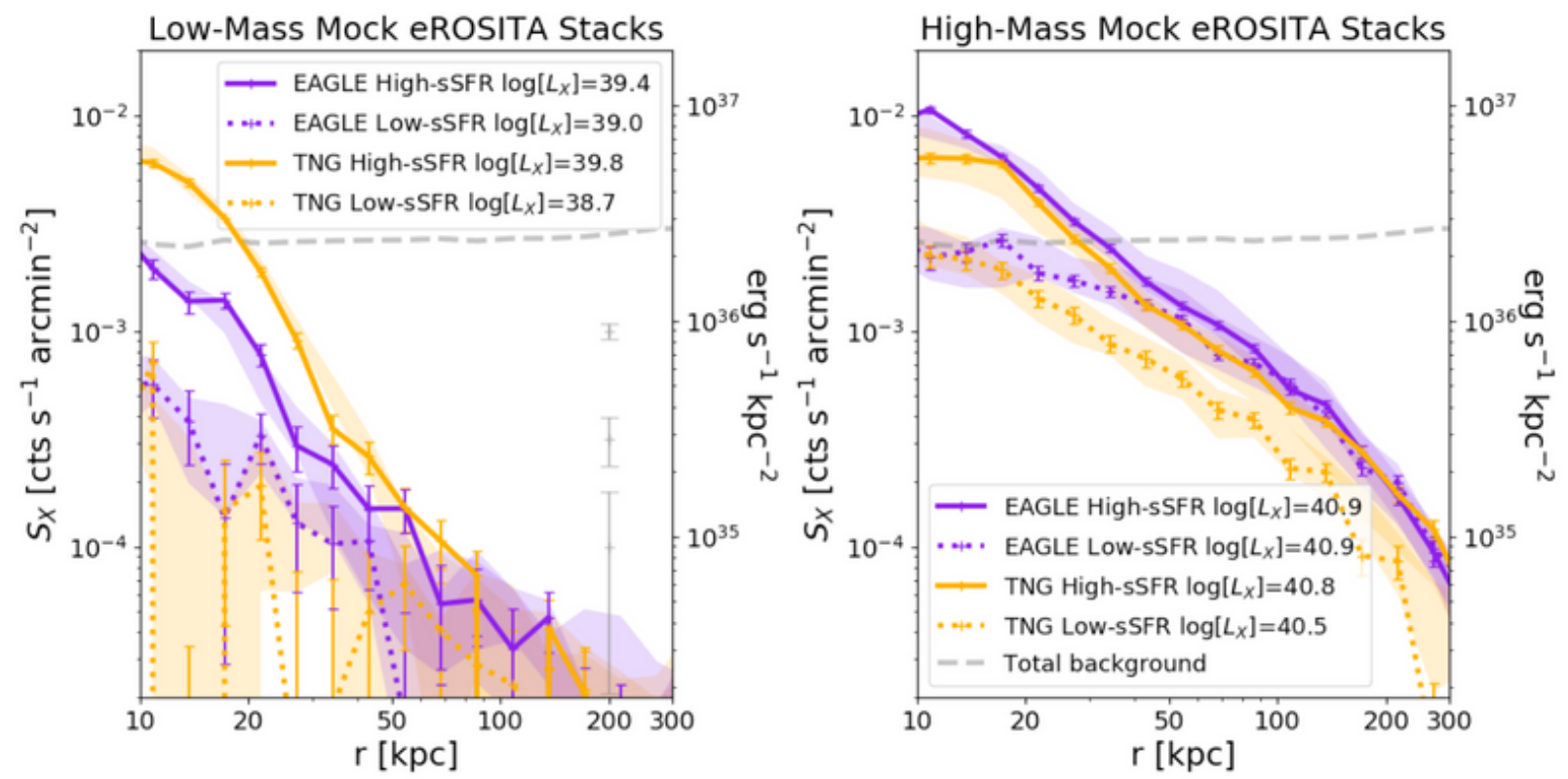

Figure 3

X-ray surface brightness profiles obtainable in principle through stacking of eROSITA data (see Oppenheimer et al. 2020 for details). On the left are stacks of $\mathbf{1 0 0}$ galaxies of total mass $\sim 10^{12} \mathrm{M}_{\odot}$ solar masses. On the right are stacks of $\mathbf{5 0}$ galaxies of total mass $\sim 10^{12.5} \mathrm{M}_{\odot}$. Predicted surface brightnesses come from the Illustris-TNG and EAGLE simulations.

Perhaps most exciting of all are the constraints that are now arriving from microwave and radio observations (e.g. [7]). The next decade promises to be a golden era in CGM studies using the Sunyaev-Zeldovich Effect. And dispersion-measure observations of Fast Radio Bursts are rapidly opening a new window on galactic atmospheres (e.g., [ㅁ]). 


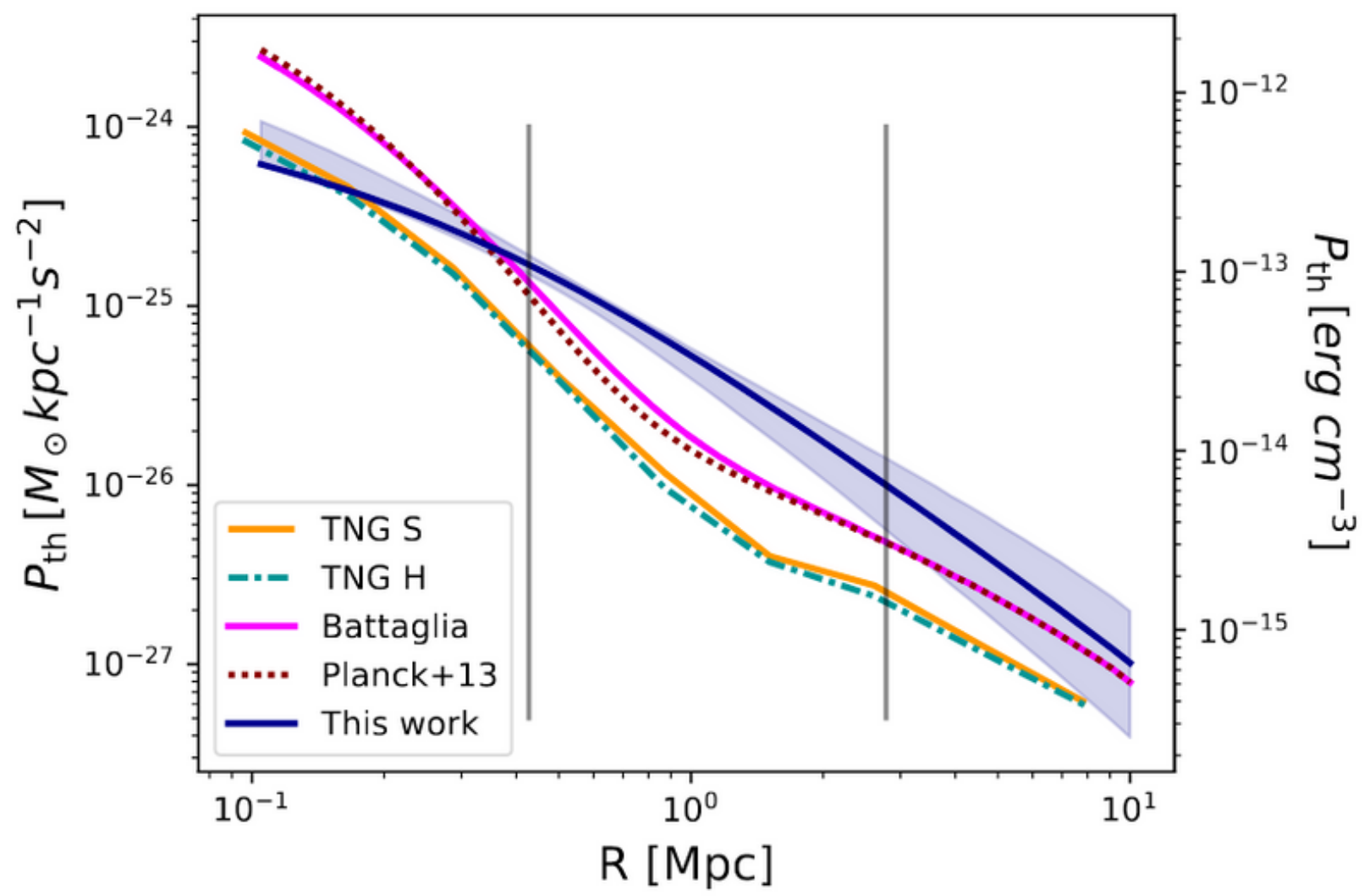

Figure 4

A circumgalactic pressure profile inferred from observations of the Sunyaev-Zeldovich Effect with the Atacama Cosmology Telescope (from Amodeo et al. 2020). They were derived from stacking observations of regions around galaxies of stellar mass $\sim 10^{11.3} \mathrm{M}_{\odot}$. A blue line indicates the best-fitting pressure profile.

At the recent workshop on Fundamentals of Gaseous Halos sponsored by the Kavli Institute for Theoretical Physics, momentum began to build around the idea of a Santa Barbara CGM Model with two primary components:

- Descriptive Parametric Models. This type of model is meant to provide a purely descriptive parameter set defining a basis for maximum-likelihood constraints. The pressure-profile model outlined above is a potential example of just one such domain.

- Heuristic Interpretive Models. Models falling into this category are inspired by particular theoretical interpretations, such as cooling flows, precipitation, or galactic winds. They can be mapped into the parameter space of descriptive models. 


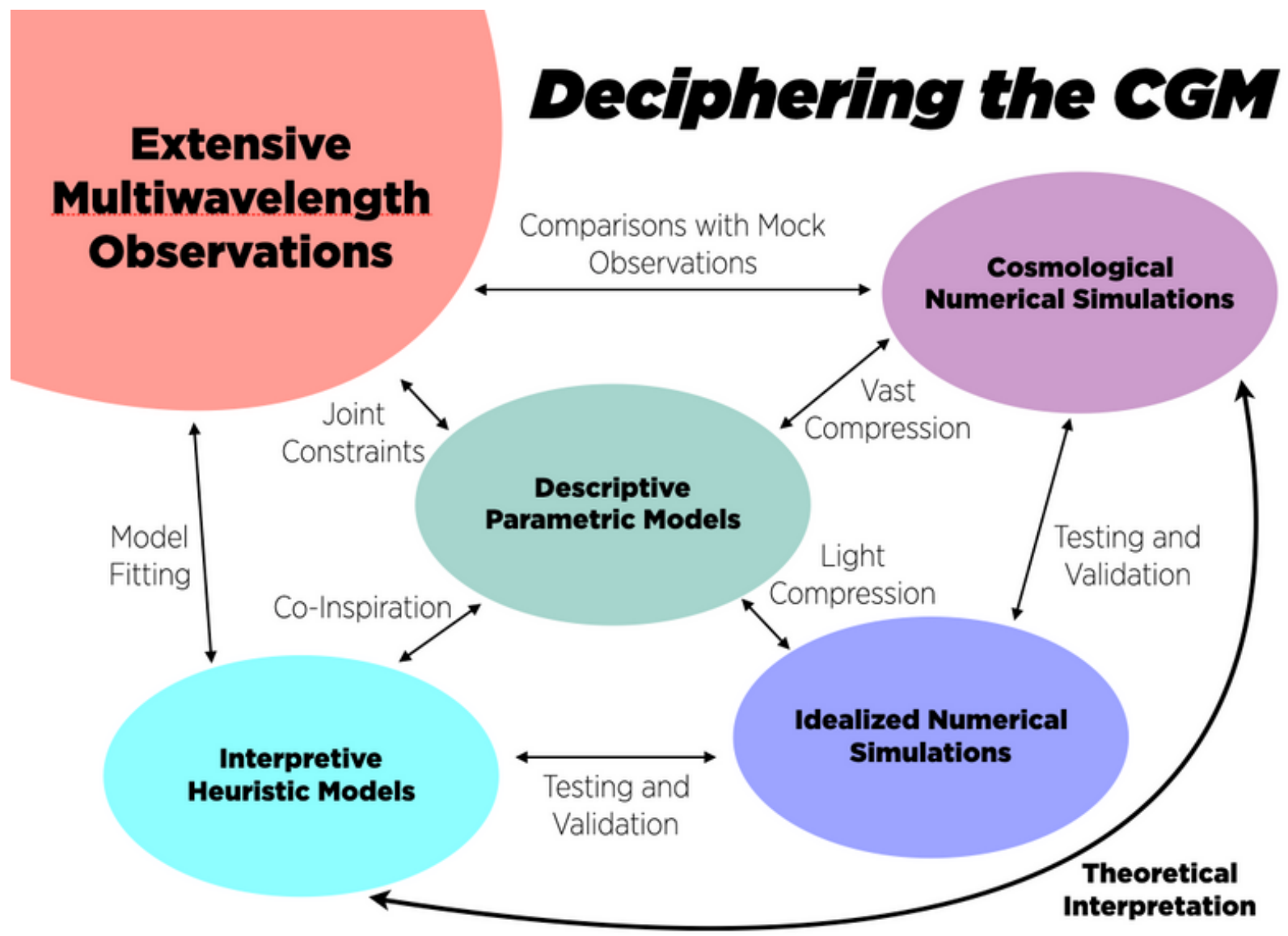

Figure 5

Conceptual diagram, originally presented at the KITP workshop on Fundamentals of Gaseous Halos, illustrating interdependent approaches for interpreting observations of the circumgalactic medium.

This new parametric modeling project was inspired by the classic Santa Barbara Cluster Comparison Project [9]. Two decades ago that consensus approach enabled productive comparisons between codes designed for cosmological hydrodynamics by defining a common set of initial conditions for the comparisons.

The most important feature of a Santa Barbara CGM Model will be broad adoption of common parameter sets for comparisons that combine complementary CGM data sets, which can also be used for comparisons between large cosmological simulations and large observational surveys.

A common language for describing the CGM helps us all, because it makes all of those comparisons much simpler. In pursuit of that goal, perfectionism needs to yield to pragmatism, as consensus and ease of use are paramount. 
We expect that the community will desire an overall parameter set far more comprehensive than the limited subdomain presented here, which describes just the CGM pressure profiles. And even that in subdomain, dependences on non-thermal pressure components, metallicity, redshift, and the spectral shape of the extragalactic UV background remain unspecified.

The Santa Barbara CGM Model project is just getting started, and we need your help. So if you're interested in participating, please contact the authors of this article.

\section{Citations}

1. Spitzer, Lyman, Jr. (1956). On a Possible Interstellar Galactic Corona. ApJ, 124, 20. https://doi.org/10.1086/146200

2. Diemer, Benedikt, More, Surhud, \& Kravtsov, Andrey V. (2013). The Pseudoevolution of Halo Mass. ApJ, 766(1), 25. https://doi.org/10.1088/0004-637X/766/1/25 $\Xi$

3. Voit, G. Mark. (2019). Ambient Column Densities of Highly Ionized Oxygen in Precipitation-limited Circumgalactic Media. ApJ, 880(2), 139.

https://doi.org/10.3847/1538-4357/ab2bfd $\leftrightharpoons$

4. Voit, G. Mark, Donahue, Megan, Zahedy, Fakhri, Chen, Hsiao-Wen, Werk, Jessica, Bryan, Greg L., \& O'Shea, Brian W. (2019). Circumgalactic Pressure Profiles Indicate Precipitation-limited Atmospheres for $\mathrm{M} \backslash$ * 109-

1011.5 \textbackslash,M\\textbackslashodot. ApJL, 879(1), L1. https://doi.org/10.3847/2041-8213/ab2766 $\triangleq$

5. Anderson, Michael E., Gaspari, Massimo, White, Simon D. M., Wang, Wenting, \& Dai, Xinyu. (2015). Unifying X-ray scaling relations from galaxies to clusters. MNRAS, 449(4), 3806-3826. https://doi.org/10.1093/mnras/stv437 6. Oppenheimer, Benjamin D., Bogdán, Ákos, Crain, Robert A., ZuHone, John A., Forman, William R., Schaye, Joop, ... Ghirardini, Vittorio. (2020). EAGLE and IllustrisTNG Predictions for Resolved eROSITA X-Ray Observations of the Circumgalactic Medium around Normal Galaxies. ApJL, 893(1), L24. https://doi.org/10.3847/2041$\underline{8213 / \mathrm{ab} 846 \mathrm{f}} \leftrightharpoons$ 7. Amodeo, Stefania, Battaglia, Nicholas, Schaan, Emmanuel, Ferraro, Simone, Moser, Emily, Aiola, Simone, ... Xu, Zhilei. (2020). The Atacama Cosmology Telescope: Modeling the Gas Thermodynamics in BOSS CMASS galaxies from 
Kinematic and Thermal Sunyaev-Zel'dovich Measurements. ArXiv E-Prints, arXiv:2009.05558.•

8. Prochaska, J. Xavier, Macquart, Jean-Pierre, McQuinn, Matthew, Simha, Sunil, Shannon, Ryan M., Day, Cherie K., ... Tejos, Nicolas. (2019). The low density and magnetization of a massive galaxy halo exposed by a fast radio burst. Science, 366(6462), 231-234. https://doi.org/10.1126/science.aay $\underline{0073} \leftrightharpoons$

9. Frenk, C. S., White, S. D. M., Bode, P., Bond, J. R., Bryan, G. L., Cen, R., ... Yepes, G. (1999). The Santa Barbara Cluster Comparison Project: A Comparison of Cosmological Hydrodynamics Solutions. ApJ , 525(2), 554-582. $\underline{\text { https://doi.org/10.1086/307908 }}$ 